\title{
Chorioretinal Folds Associated With Different Etiologies
}

\author{
Tuncay Topal* and Eyüp Düzgün \\ Department of Ophthalmology, Karabük, Turkey
}

Received: February 13, 2017; Published: February 21, 2018

*Corresponding author: Tuncay Topal, Department of Ophthalmology, Safranbolu, Turkey, Tel: 90541 2718788; Email: drtuncaytopal@gmail.com

\begin{abstract}
Chorioretinal folds are parallel grooves or striae involving the inner choroid, Bruch membrane, the retinal pigment epithelium and sometimes the retina. In the current study, our purpose is to present clinical and imaging study findings of five patients we have diagnosed chorio retinal folds seen together with hypotonia maculopathy, acquired hyperopia, angioid streaks, diabetic retinopathy and agerelated macular degeneration.
\end{abstract}

Keywords: Choroidalfold; Retina; Bruch's Membrane; Retinal Pigment Epithelium

Abbreviations: CF: Chorioretinal Folds; RPE: Retinal Pigment Epithelium; FFA: Funds Fluoresce in Angiography; CNV: Choroidal Neo Vascular; IOP: Intra Ocular Pressure; OCT: Optical Coherence Tomography; VA: Visual Acuity; UBM: Ultrasonic Bio Microscopy; FAF: Funds Auto Fluorescence; MP: Micro Perimetry; BCVA: Best Corrected Visual Acuity

\section{Introduction}

Chorioretinal folds (CFs) are most often fluctuating or streaking, which is also found in the posterior pole, which can also include internal choroid, Bruch's membrane, retinal pigment epithelium (RPE) and occasionally neurosensorial retinas. A series of thin lines or strikes, usually arranged in light-dark color that extends parallel to each other but can be more or less vertical or oblique and very rarely extends beyond the equator.CFs were first described by Edward Nettle ship in a patient with atrophic papilledema due to an intracranial mass in 1884 [1]. The yellow, hypo pigmented overhangs are the areas where the RPE is stretched and tapered, while the darker and deeper bands are the areas where the RPE is under compression. This appearance of RPE is also the cause of hyper and hypolorescence in funds fluoresce in angiography (FFA). In 1972, Newell stated that the CFs was formed due to the close connections between the Bruch membrane and the underlying choir-capillaries [2].

When the choroid swells and expands, the upper Bruch membrane becomes forced to fold and the clinical appearance of the CFs is formed. Idiopathic choriretinal folds associated with benign conditions such as hypermetropia are the most common, but the orbital mass, inflammation, sclerotic, sclera buckling, choroid mass, choroidalneovascular membrane (CNV), chronic papilloma, central serous chorioretinopathy (SSCR), thyroid eye disease, uveal effusion syndrome, age-related macular degeneration, trauma, drug use, and hypotonic have been reported [3-12]. Idiopathic CFs is diagnosed when no etiologic factor is detected. Systemic research is essential for the identification of a specific etiology if folds are seen on the funds examination. Patients may be asymptomatic, as well as with hyperopic or metamorphosis. In this article, we aimed to present a series of five cases of CFs with different etiologies.

\section{Case 1}

A 21-year-old male patient was referred due to low vision and intraocular pressure (IOP) in the right eye. The patient stated that he had been wounded with a wire in the right eye about thirty days ago and cataract developed because of the trauma. He stated that IOP was low during the postoperative examinations and that he was referred for this reason. In the right eye; the visual acuity (VA) was $6 / 10$ with correction of +3.00 diaper (D) on the right eye, the IOP was $4 \mathrm{~mm} \mathrm{Hg}$, biomicroscopic examination revealed a penetrating line (Seidel negative), which was sutured to the temporal limbos and pseudo phobia, funds examination revealed a tortuosity increase in all vascular structures starting from optic disc, wrinkles and mauls in the per papillary region were observed. The left eye was completely normal. Axial length measurements performed with A-mode ultra sonography showed 22,19 $\mathrm{mm}$ in the right and 22,70 $\mathrm{mm}$ in the left eye. Optical coherence tomography (OCT), ultrasonic biomicroscopy (UBM) and funds auto fluorescence (FOF) images were obtained.

As a result of the examinations, traumatic cyclodialysis induced hypotoniamaculopathy was diagnosed for right eye. Topical 
corticosteroid and cyclopentolate therapy was initiated, and 3 days later the right eye axis was measured as 22,80 and the refraction rate was measured as $+0,75$. The IOP returned to $18 \mathrm{mmHg}$ and the VA was 10/10. Comparing the OCT images taken at the end of treatment with the first received OCT, the choroid, RPE and wavy appearance of the retina (Figure 1a). Were seen to be healed at the end of treatment (Figure 1b). Cyclodialysis was detected in the temporal quadrant (Figure 2a). By UBM and normal culinary anatomy was obtained at the end of the treatment (Figure $2 b$ ). There was a normal appearance at the end of the treatment (Figure 3a), while significant increase in tortuosity was observed in vascular structures at the first examination of the FOF (Figure 3b).

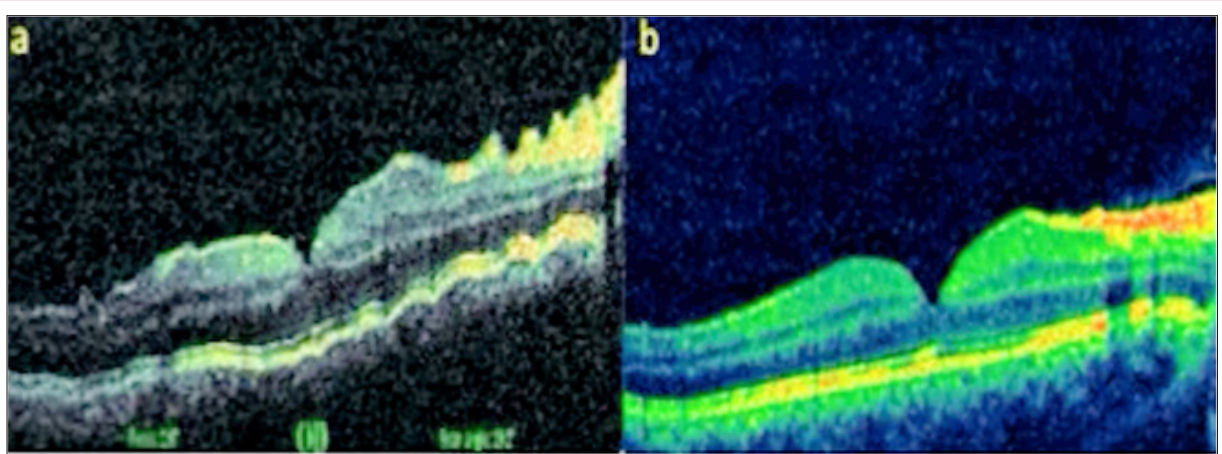

Figure 1: Right eye view (a) at first examination and (b) post-treatment OCT images.

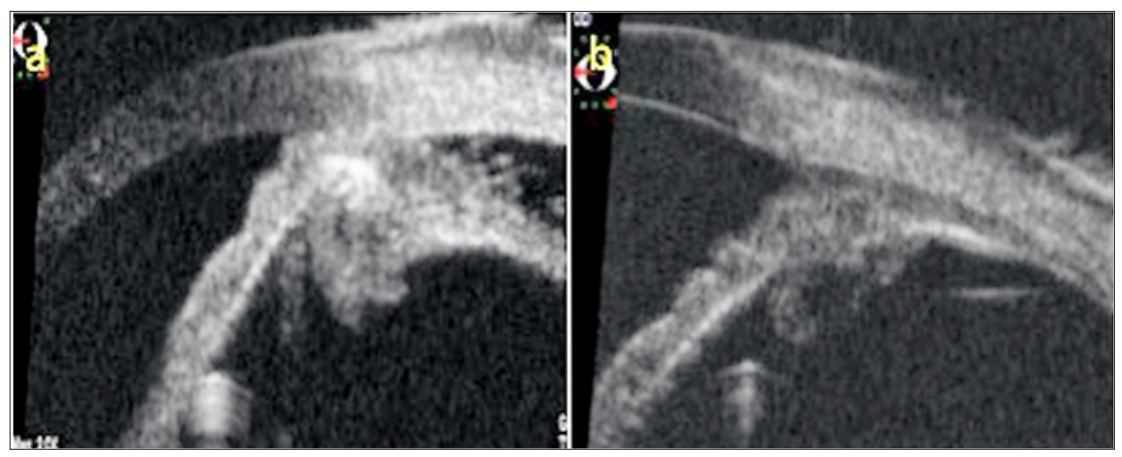

Figure 2: a. Temporal quadrant UBM images of the right eye at first and b. At the end of treatment.

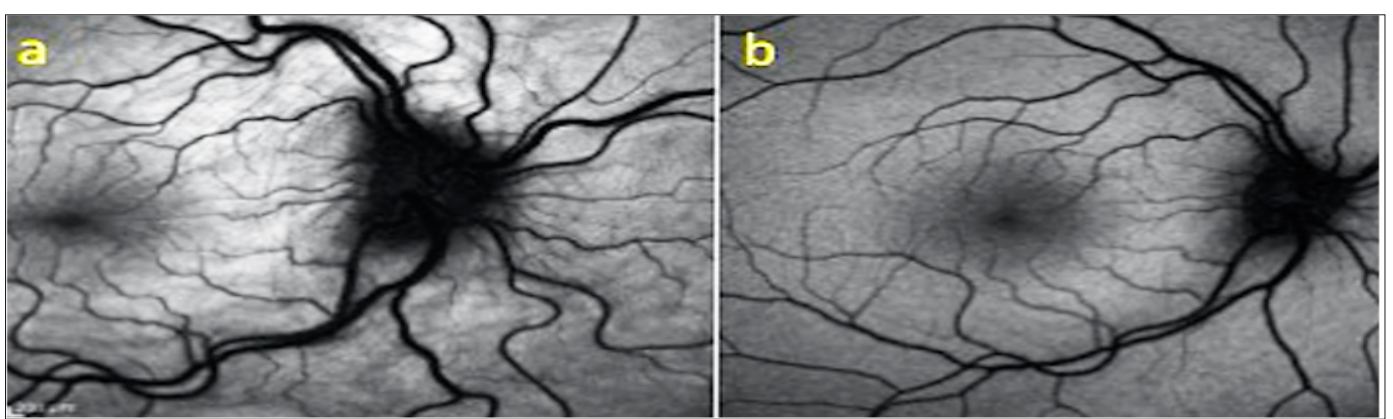

Figure 3: a. Right-eye at first and treatment outcome, b. FOF images taken at $30^{\circ}$ magnification.

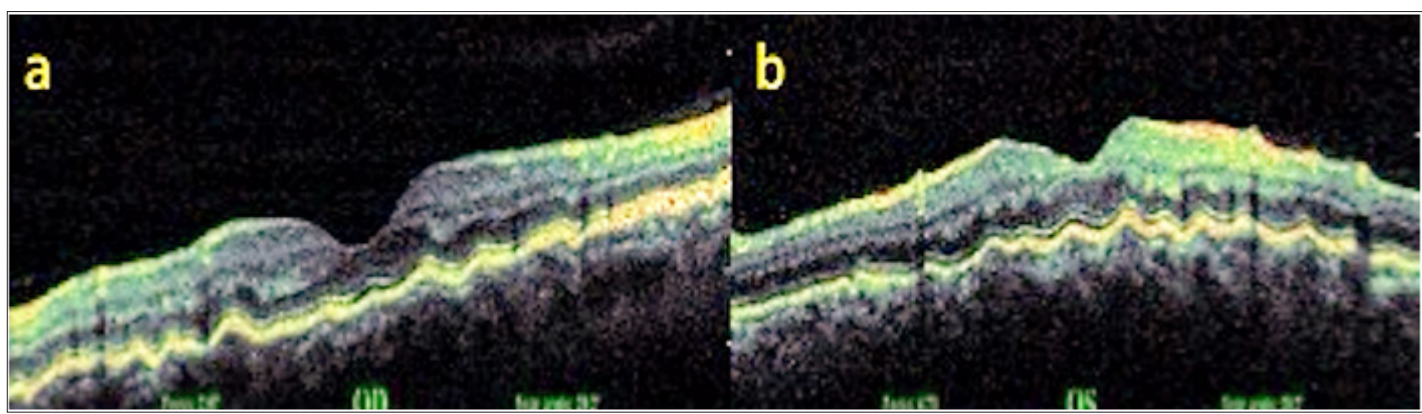

Figure 4: OCT images $(a, b)$. 


\section{Case 2}

A 53-year-old male presented with complaints of blurred vision. Patient who did not use glasses and had uncorrected VA were 0,7 in both eyes, with $+3.00 \mathrm{D}$ correction in both eyes the Varies up $10 / 10$, and the IOP was $15 \mathrm{mmHg}$ in both eyes. Anterior segment biomicroscopic examination was normal in both eyes. Light-dark horizontal lines extending from the optic disc to the macula were observed in both eyes in the funds examination. In OCT, CFs was observed in choroid, RPE and neurosensory layers in both eyes (Figures 4a \& 4b). The patient's FFA did not reveal any features other than CFs. Radiologic imaging studies for orbital, intracranial, and systemic pathologies were performed and the patient was diagnosed as CFs associated with an acute acquired hyperopic.
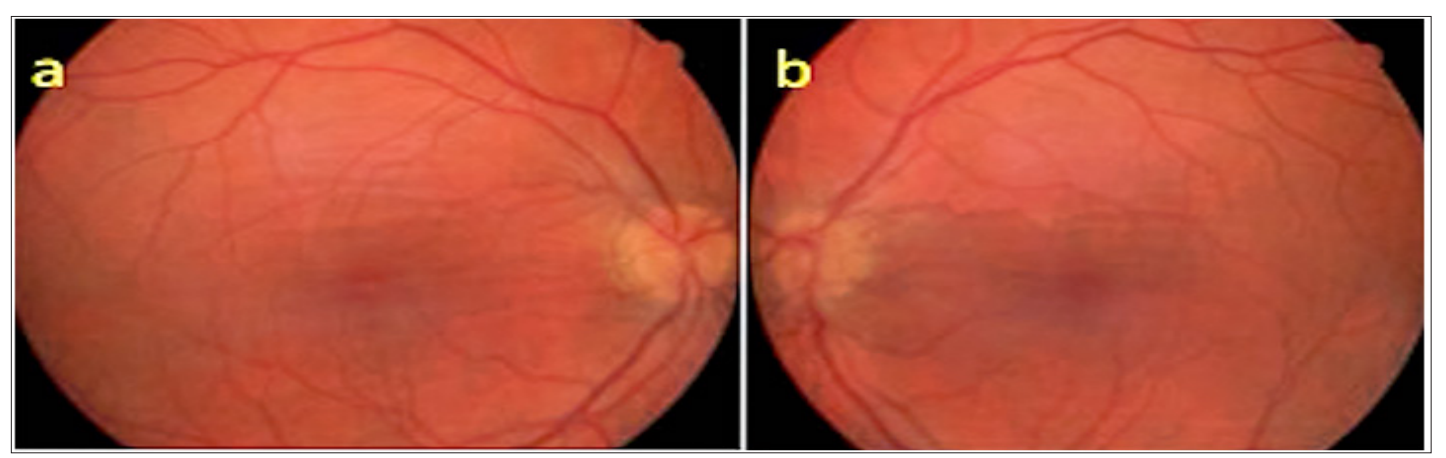

Figure 5: Fundus appearance in both eyes $(a, b)$.

\section{Case 3}

A 50 year old male presented with complaints of blurred vision. The VA was 9/10 in both eyes with no refractive correction need. Anterior segment biomicroscopic examinations were of course normal in both eyes. IOPs were $14 \mathrm{mmHg}$ in both eyes. In the fundus examination, several angioid streaks and light-dark colored bands were seen in the per papillary region in both eyes (Figure $5 a \& 5 b$ ). Wrinkles were observed in choroid, RPE and neurosensory layers in
OCT (Figure 6a \& 6b). In FFA, more prominent angioid streaks were observed around the optic disc in the left eye, and hyper fluorescence due to RPE cracks was observed (Figure 7a \& 7b). Microperimetry (MP) revealed that sensitivity was normal in folding areas while sensitivity was decreased in areaswith RPE defect (Figure 8a \& 8b). Radiologic imaging studies and laboratory examinations for orbital, intracranial and systemic pathologies were within normal limits. CFs associated with angioid streaks was diagnosed.

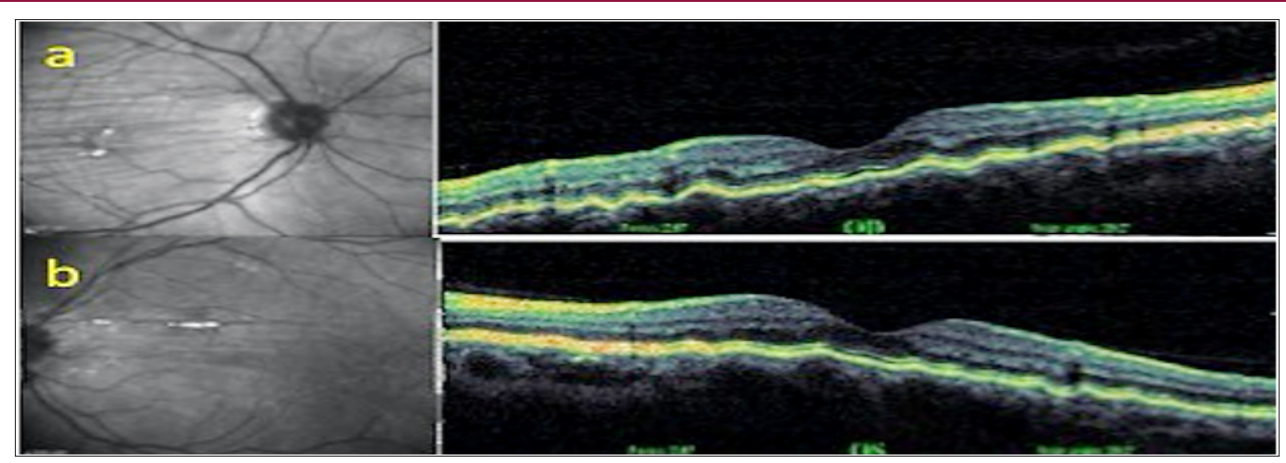

Figure 6: OKT images $(a, b)$.
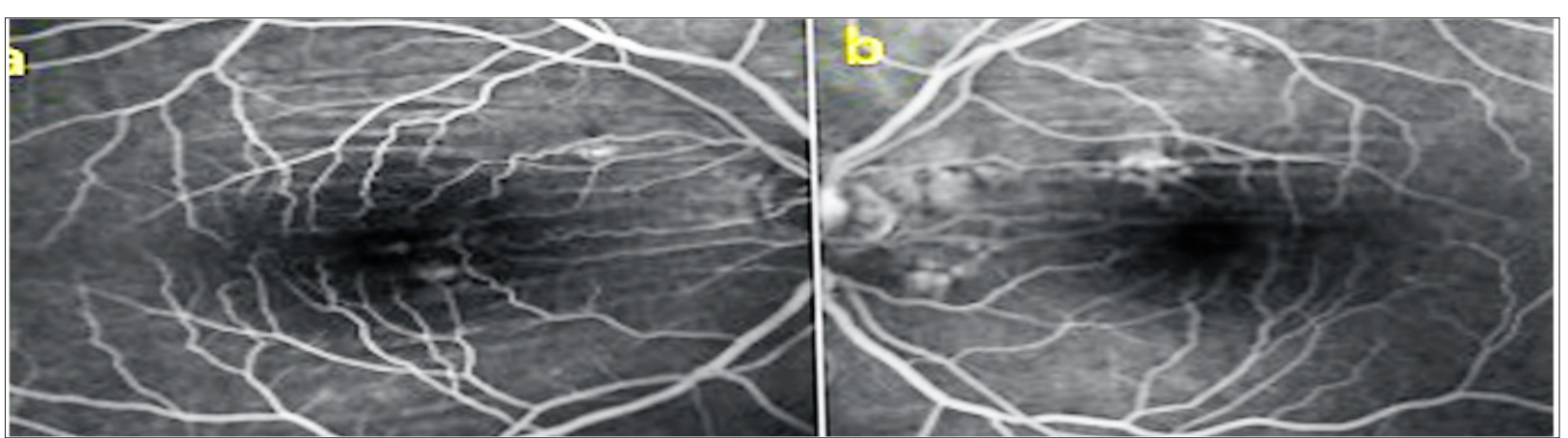

Figure 7: FFA images $(a, b)$. 


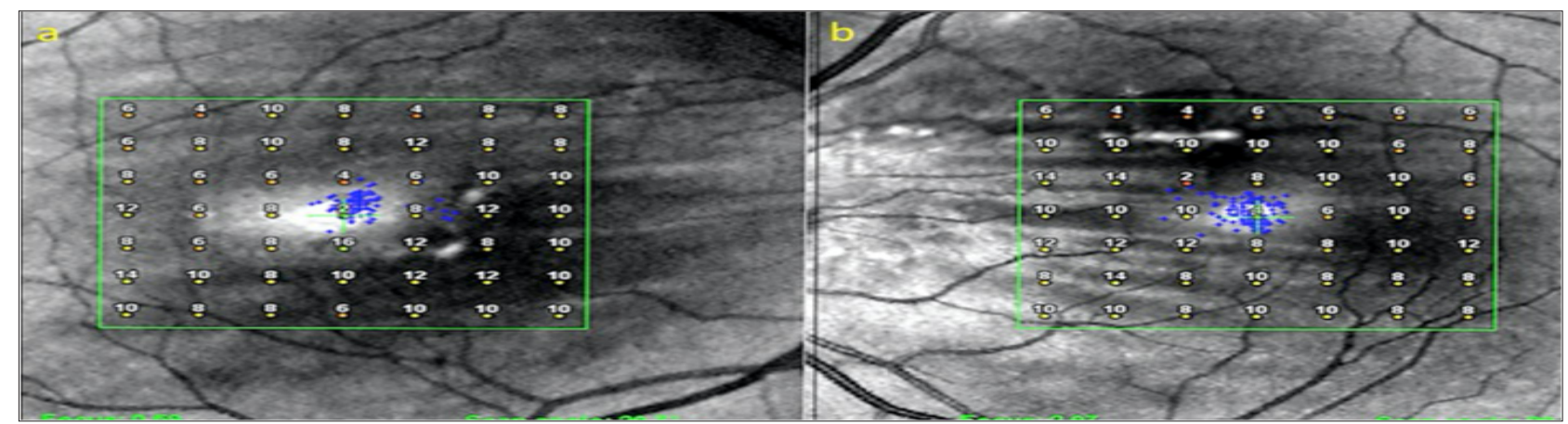

Figure 8: Microperimetry $(a, b)$.

\section{Case 4}

During the follow-up of an 81-year-old diabetic female, oblique choroid folds were detected outside the vascular arquades in both eyes. The VA was 8/10 in the right eye and 9/10 in the left eye. In biomicroscopic examination, posterior chamber intraocular lens was present in both eyes and IOPs were $13 \mathrm{mmHg}$ bilaterally. In the fundus examination, moderate diabetic retinopathy findings and CFs were observed in both eyes. In OCT, RPE was observed to be fluctuating in the cross sections of the superior vasculature (Figure 9a \& 9b). In FFA, CFs were observed in both eyes as light-dark lines with more obvious oblique outcrops than the upper main vascular arquade, and there were micro aneurysms occasionally due to diabetic retinopathy (Figure 10a \& 10b).
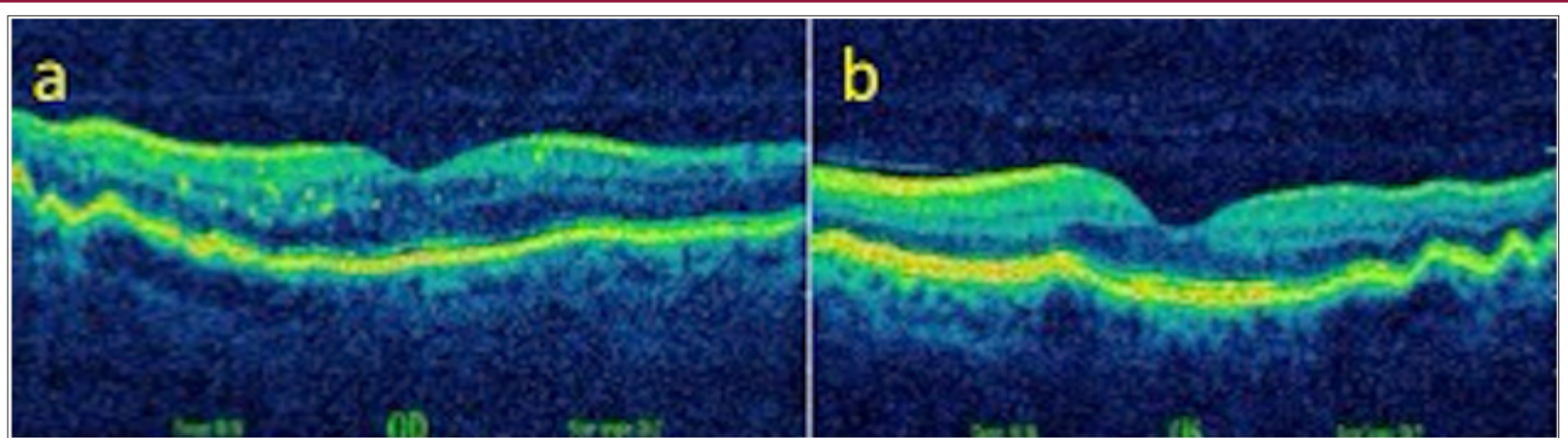

Figure 9: OKT images $(a, b)$.

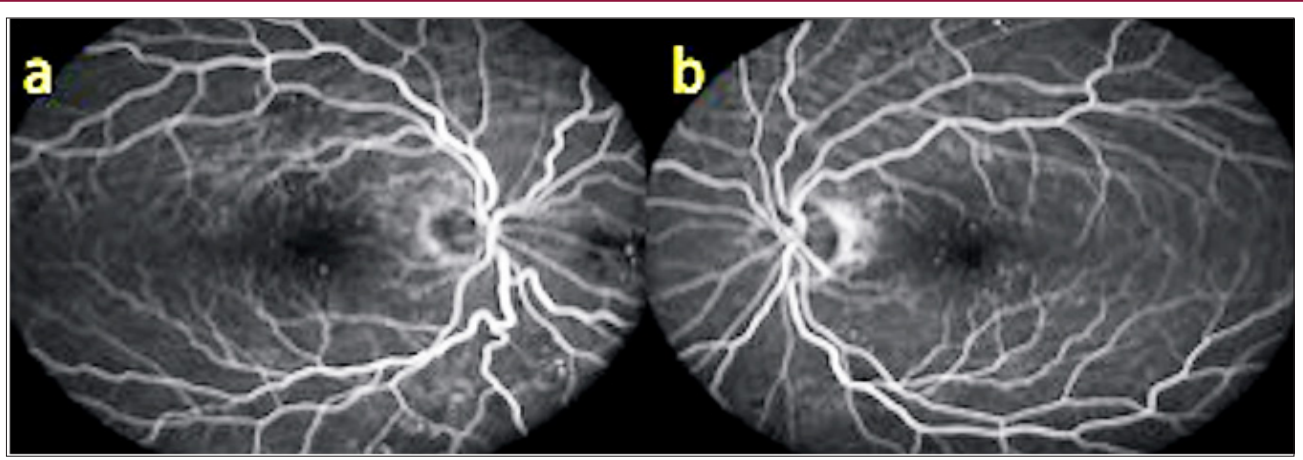

Figure 10: FFA images $(a, b)$.

\section{Case 5}

An 81-year-old male who was followed up for age-related macular degeneration, horizontal-extending $\mathrm{CFs}$ were noted in the posterior pole of the left eye. VA was in the right eye at the level of hand movement perception and at the level of $7 / 10$ in the left eye. Biomicroscopic examination revealed pseudophakia in both eyes. IOPs were $15 \mathrm{mmHg}$ in both eyes. Inthefundusexamination, peripapillaryatrophy, macularscarand RPE detachmentareseen in therighteye (Figure 11a), withlight- darkbanding in theuppervascularquadrant, especially in thelefteye (Figure 11b). In the right eye, wide RPE detachment and sub retinal fluid were observed in the right eye (Figure 12a), while choroid and RPE wrinkles were observed in the left eye (Figure 12b). FFA revealed age-related neo vascular macular degeneration (Figure 13a). Horizontal extension of theCFstotheupperhalf of themaculaandveinligationaroundtheopticdiscwereobserved in the left eye (Figure 13b). Intravitreal ranibizumab therapy for the right eye of the patient was continued while left eye was taken in the direction of the CFs. 


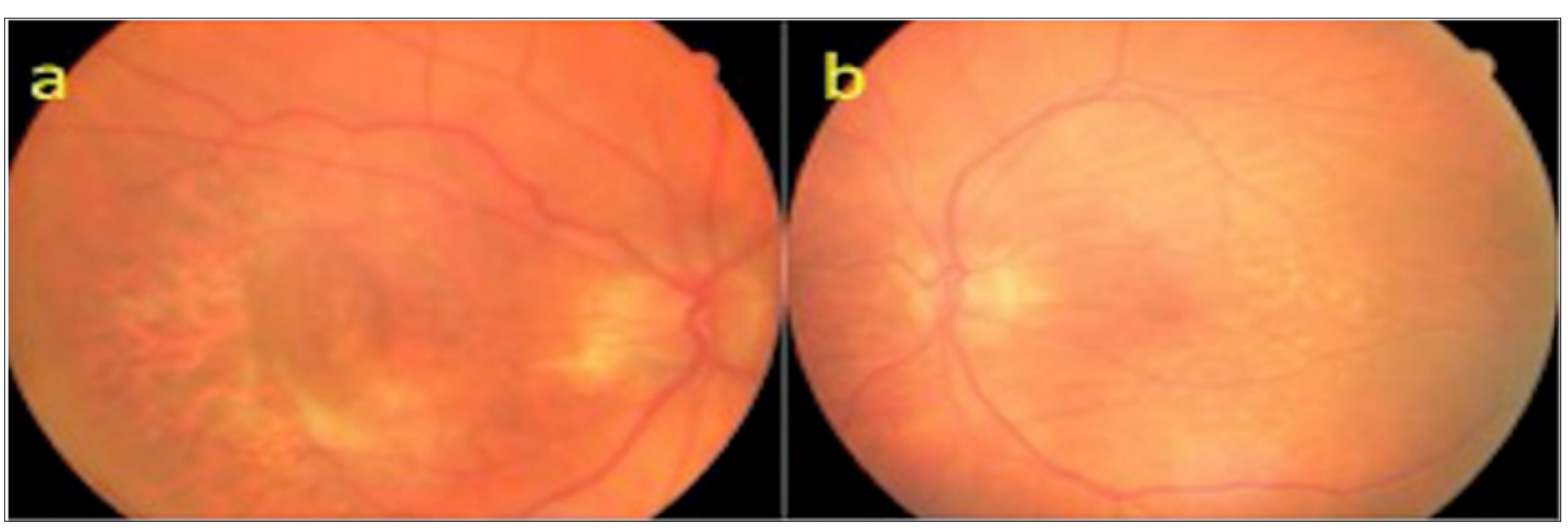

Figure 11: Fundus appearances $(a, b)$.

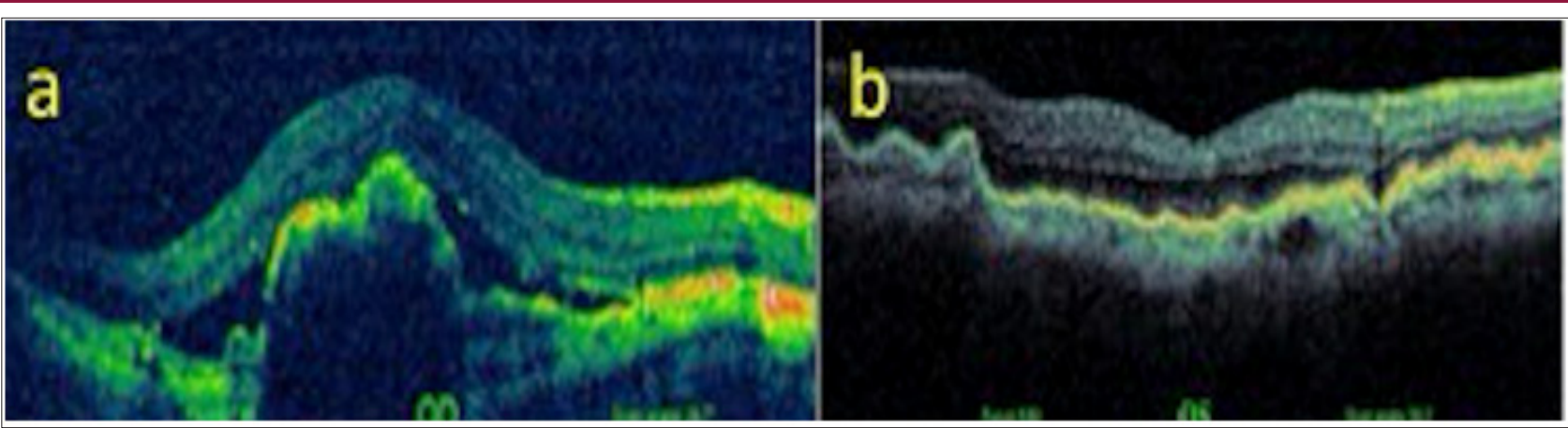

Figure 12: OCT images $(a, b)$.

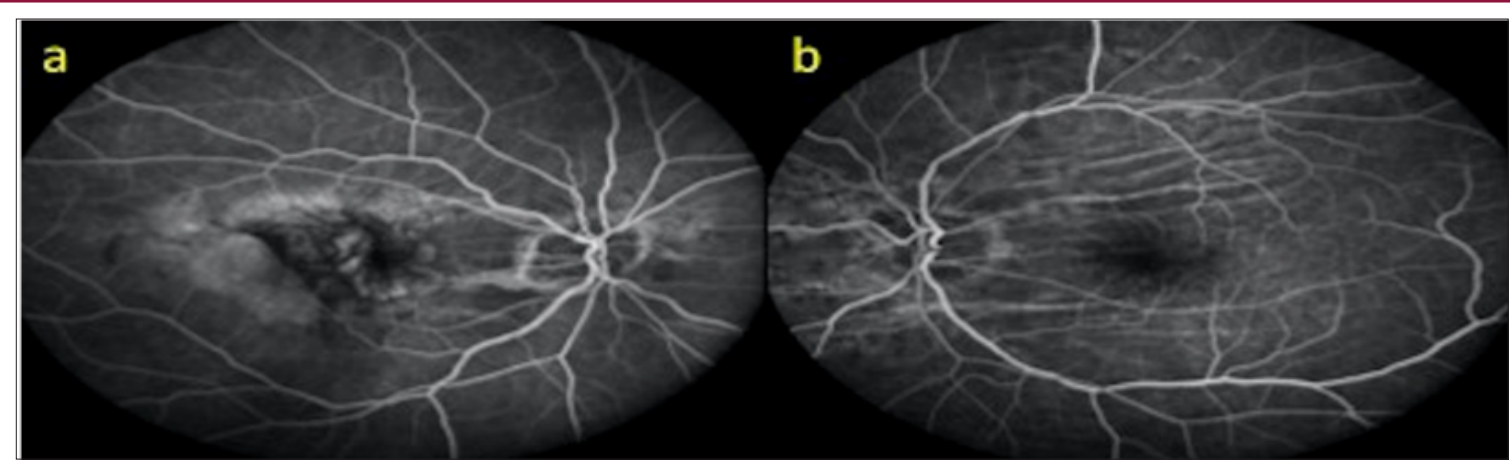

Figure 13: FFA images $(a, b)$.

\section{Discussion}

CFs is not common, but they are easy to diagnose when they are seen on funds examination because of their characteristic appearance. Clinically, the posterior pole is usually seen as diffuse light-dark banding. CFs may be parallel, radial or randomly distributed. Patients may be asymptomatic or may be presenting with metamorphosis or hypermetropia. The cause of visual impairment is distortion of the overlying photoreceptor layer, but in chronic cases the neurosensory retina and RPE may develop permanent damage. If it can be detected, treatment is directed to etiology. According to the currently accepted view, any intraocular or extrinsic event that disrupts the anatomical relationship of sclera with choroid and retina may cause CFs by affecting choroid, Bruch's membrane, RPE and neurosensory layer. The CF is a kind of compensator response given to the disruption of the normal spherical shape of the globe. Clinical examination is sufficient for the diagnosis of CFs, but FFA is the gold standard imaging technique.

Lately it has become easier to diagnose, thanks to OCT, which is increasingly used and allows visualization of the cross-sectional anatomy of the choroid and retinas. Another non-invasive imaging modality, B-mode USG, augments the etiologic investigation by showing thickening of the sclera and choroids, especially during inflammation and mass-induced tumors [13]. We also found that the UBM which we used in our first case and demonstrated cyclodialysis is invaluable in that it provides us with the opportunity to assess the anatomy of the anterior segment, is a non-invasive imaging method. As in our case, idiopathic CFs are usually bilateral in healthy hypermetropic individuals and best corrected visual acuity (BCVA) is preserved. Choroid tumours (melanocytes, choroid hemangiomas and stomas), mainly melanomas, and metastases 
from organs such as the lungs and breast may be the cause of CFs. Regardless of whether it is malignant or benign, they may cause IOP riseand may result in CFs.

Cavernous hemangioma, lymphangioma, hemangiopericytoma, gloom, meningioma, rhabdomyo sarcoma, lymphoma, fibrous histiocytoma, epithelial tumors of lacrimal gland, neuroblastoma and metastatic tumors are the examples. Intraconal masses of these types can cause hypermetropia and the extracoronal ones induce astigmatism and cause refraction error. The collapsing materials used in the treatment of retinal detachment, radiotherapy implants, and implants used for orbital fractures may also cause CFs through globe compression [13]. Autoimmune connective tissue diseases such as posterior sclerotic, orbital inflammatory pseudo tumor, thyroid eye disease, rheumatoid arthritis, vacuities such as Wegener and Poliarteritisnodosa (PAN) are causes of inflammation-induced Cling. These patients may also present with pain, tenderness, proptosis, and lack of vision. It is noted in the literature that selflimiting CFs may develop after local supracoroidal hematomas due to mass effect when it is not even solid [14].

CFs can be seen in the hypotoniamaculopathy caused by wound infiltration, excessive filtration after glaucoma surgery, and cyclodialysis. As seen in the first case, edema-like appearance in the optic disc and engorgement in vascular structures are noteworthy findings. With surgery for etiology or medical treatment as in our case, the funds appearance usually returns to normal and visual acuity increases, but after extended hypo tony, the folding may persist. As an example of postoperative CFs in the literature, Liu et al. with 25 gauge Trans conjunctives alsutureless vitrectomy for recurrent epiretinal membrane showed choroid detachment and CFs in a patient with hypo tony. After 3 months of treatment, the folds persisted and the VA increased to 20/40 from the hand movements [15]. Choroidalneovascular membrane due to agerelated macular degeneration, spontaneously or through postlaser contraction, may cause CFs. Intracranial masses, pseudo Tumorcerebri, benign intracranial hypertension and papilledema due to duralarteriovenous malformation may also be the cause of CFs. CFs may persist after papilledema treatment, but VA is rarely affected.

Talban et al. showed an increase in BCVA despite the persistence of CFs after tumor excision in a patient with parasellar meningioma [16]. Choroidal fold progression due to globe compression was reported due to medial wall injury of the orbital wall during nasal polypectomy [17]. It has been reported that in a patient with persistent chorioretinal adhesion due to trauma, the systemic steroid response was not available and that after 12 months the intravitreal injection of triamcinolone acetonide resulted in a level of VA of 0.2 [18]. CFs has been reported with pseudo vitally for Mmaculopathy, nanoftalmus, and micro phthalmus [19-21]. It has been reported in Vogt Koyanagai Harada's syndrome, and even in the literature that this exacerbation period of the syndrome may be a leading indicator [22]. In some patients using topiramate, it has been reported that reversible macular folds can be the observed [23]. In diabetic retinopathy cases, some CF cases have been reported. While CFs can be seen in diverse stages of diabetic retinopathy, they are usually asymptomatic, with symmetrical, macular, varying lengths.

The relationship between diabetic retinopathy and CFs is not well defined, and it is thought that about half of the cases can be seen in diabetic patients with increased arterial hypertension and increased choroidal thickness [24,25]. As a result, one of the five CFs was evaluated as traumatic hypotonic, one was associated with acute acquired hyperopic, one was associated with algoid streaks, one was associated with diabetic retinopathy, and one was associated with age-related macular degeneration. As seen in the literature and in our cases, the CFs which will keep the idiopathic cases out is not a primary pathology but rather a compensatory response given to many pathologic conditions which may or may not be related to the eye but distort the spherical shape of the eye. In such cases, detection of etiologic factor is important because it will determine the treatment. Every ophthalmologist should be aware of orbital or systemic pathologies that may cause chorioretinal folding. A careful history should be taken and medical history, inflammatory, euplastic, infectious and infiltrative diseases should be questioned diligently.

\section{References}

1. Nettleship E (1884) Peculiar lines in the choroid in a case of post papilliti catrophy. Trans Ophthalmol Soc 4: 167-168.

2. Newell FW (1973) Choroidal folds. The seventh Harry Searls Gradle Memoriallecture. Am J Ophthalmol 75(6): 930-942.

3. Norton EWD (1969) A characteristic fluoresce in angiographic pattern in choroidal folds. Proc R SocMed 62: 119-128.

4. Kroll AJ (1970) Norton EWD. Regression of choroidal folds. Trans Am Acad Ophthalmol Otolaryngol 74(3): 515-5126.

5. Hyvarinen L, Walsh FB (1970) Benign chorioretinal folds. Am J Ophthalmol 70(1): 14-17.

6. Cangemi FE, Trempe CL (1978) Walsh JB. Choroidal folds. Am J Ophthalmol 86(3): 380-387.

7. Kalina RE, Mills RP (1980) Acquire dhyperopia with choroidal folds. Ophthalmology 87(1): 44-50.

8. Friberg TR, Grove AS (1983) Choroidal folds and refractive errors associated with or bitaltumors An analysis. Arch Ophthalmol 101: 598603.

9. Newell FW (1984) Funduschanges in persistent and recurrent choroidal folds. Br J Ophthalmol 68(1): 32-35.

10. Leahey AB, Brucker AJ, Wyszynski RE, Shaman PS (1993) Chorioretinal folds. A comparison of unil at eraland bilateral cases. ArchOphthalmol 111(3): 357-359.

11. Kook Young Kim, Hyung WooKwak, Moosang Kim, Seung Young Yu (2013) Traumatic chorioretinal folds treated with intra-vitrealtriam cinolone injection. Indian J Ophthalmol 61(4): 179-182.

12. William Gualtieri, Jan Janula (2013) Topirama temaculopathy. IntOphthalmol 33(1): 103-106.

13. Gass JDM (1987) Folds of the choroidand retina. In Stereoscopic Atlas of Macular Diseases, $3^{\text {rd }}$ (Edn.). In: MO Mosby, St Louis (Eds.). USA, pp. 221-234.

14. Augsburger JJ, Coats TD, Lauritzen K (1990) Localizedsuprachoroidalhematomas. Ophthalmoscopic features, fluore sceinangiography, and clinical course. Arch Ophthalmol 108(7): 968-72. 
15. Liu DTL, Chan CKM, Fan DSP, SW Lam, Lam DSC, et al. (2005) Choroidal folds after 25 gauge trans conjunctival suture less vitrectomy. Eye 19: 825-827.

16. Taban M, Kosmorsky GS, Singh AD, Sears JE (2007) Choroidal folds secondary to parasell armeningioma. Eye 21: 147-150.

17. Sevim Kavuncu, Ömür Ersoy Demirtaş, Mehmet Yasin Teke, Mahmut Demirtaş, Gültekin Köklü (2009) Nazal polipektomi sonrası koroidal fold gelişimi. Ret Vit 17: 291-294.

18. Kim KY, Kwak HW, Kim M, Yu SY (2013) Traumatic chorioretinal folds treated with intra vitreal triamcinolone injection. Indian J Oplhthalmol 61: 179-182.

19. Pardo Munoz MA, Cabrejaz Martinez L, Losada Mayo D (2012) Pseudovitelliformmacul opathyandbilateral choroidal folds: differential diagnosis. Arch Soc Esp Oftalmol 87(6): 187-190.

20. Yalcındağ FN, Atilla H, Batığlu F (2011) Optical coherencet omography findings of retinalfolds in nonophthalmos. Case Rep Ophthalmol Med 2011: 491894.

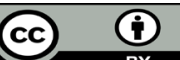

This work is licensed under Creative Commons Attribution 4.0 License

Submission Link: http://biomedres.us/submit-manuscript.php
21. Novelli FJ, Nobreqa MJ, Rosa EL, Bortolotto CM, Kuntz J (2009) Posterior segment changes in microphthalmiceyes. Arq Bras Oftalmol 72(5): 697699.

22. Minoru Tanigawa, Harutuki Ochiai, Yoko Tsukahara, Yuko Ochiai, Hiromitsu Yamanaka (2012) Choroidalfolds in acutestage VogtKoyanagi Harada Disease Patients with relatively Short Axial Length. Case Rep Ophthalmol 3(1): 38-45.

23. Kumar M, Kesarvani S, Rao A, Garnaik A (2012) Macularfolds: an unusualassociation in topiramatetoxicity. Clin Exp Optom 95(4): 449452.

24. Fagundez Vargas MA, Jimenes Parras R, Bermudez Uria L (2000) Choroidalfolds in diabetic retinopathy. Arc Soc Esp Oftalmol 75(12): 797-802.

25. Manaviat MR, Salimpour S (2013) Bilateral choroidal folds in a diabeticpatient. Iranian Journal of Diabetesand Obesity 5(4): 176-180.

\begin{tabular}{ll} 
BIOMEDICAL & Assets of Publishing with us \\
RESEARCHES & - Global archiving of articles \\
\hline ISSN: $2574-1241$ & Immediate, unrestricted online access \\
\hline
\end{tabular}

